\title{
METHOD FOR AGGREGATING CORRELATED INTERVAL GREY LINGUISTIC VARIABLES AND ITS APPLICATION TO DECISION MAKING
}

\author{
Nian ZHANG \\ School of Economics and Management, Southwest Jiaotong University, \\ Chengdu, 610031 Sichuan, China
}

Received 01 May 2011; accepted 21 August 2011

\begin{abstract}
With respect to multiple attribute decision making (MADM) problems in which attribute values take the form of interval grey linguistic variables, a new decision making analysis method is developed. In this paper, we propose the interval grey linguistic variables ordered weighted aggregation (IGLOWA) operator, and then use the Choquet integral to develop the interval grey linguistic correlated ordered arithmetic aggregation (IGLCOA) operator and the interval grey linguistic correlated ordered geometric aggregation (IGLCOGA) operator. Those operators not only consider the importance of the elements, but also can reflect the correlations among the elements. Then, we develop an approach to multiple attribute decision making problems with correlative weights which attribute values are given in terms of interval grey linguistic variables information based on those operators. Finally an illustrative example is given to use the method in the range of uncertain multiple attribute decision making. The results show that the method proposed in this paper is feasible.
\end{abstract}

Keywords: Choquet integral, multiple attribute decision making problems, interval grey linguistic variables, interval grey linguistic correlated ordered arithmetic aggregation (IGLCOA) operator, interval grey linguistic correlated ordered geometric aggregation (IGLCOGA) operator.

Reference to this paper should be made as follows: Zhang, N. 2013. Method for aggregating correlated interval grey linguistic variables and its application to decision making, Technological and Economic Development of Economy 19(2): 189-202.

JEL classification: C02, C44, C61, D70, D81.

Corresponding E-mail:

chinazhangnian@163.com 


\section{Introduction}

Since Zadeh (1965) introduced the concept of the fuzzy set and Deng (1982) firstly presented the grey system theory, these were well applied in multiple attribute decision making (Bu, Zhang 2002; Chen 1994; Jin, Lou 2004; Luo, Liu 2004; Liu, Jin 2010; Meng et al. 2007; Merigó 2010; Merigó, Gil-Lafuente 2011; Wang, Klir 1992; Wang et al. 1996; Wei et al. 2011; Xu 2010; Zhang, Liu 2010; Li 2012; Salmeron, Gutierrez 2012; Yin 2012; Zhu, Hipel 2012; Baležentis, Zeng 2013). Multiple attribute decision making (MADM) has been extensively applied to various areas such as society, economics, management, military, etc. It is well known that the object things are complex and uncertain and Human thinking is ambiguous. Hence, the majority of multiple attribute decision making is also uncertain and fuzzy, and fuzziness is the major factor in the process of decision making. However, in dealing with the problem of incomplete information caused by poor information, decision-making also demonstrates its greyness. The "fuzzy" means those uncertain factors in the evaluation information which are caused by the fuzziness of human thinking, while the "grey" means that objective uncertainty is caused by the insufficient and incomplete information. Therefore, the "fuzzy" and the "grey" are different concepts, many scholars have studied on the grey fuzzy multiple attribute decision making, which demonstrates not only its fuzziness, but also its greyness.

The research on the grey fuzzy decision making has been widely investigated and applied to a variety of fields. Chen (1994) introduced the concept of the grey fuzzy in detail in his book. Bu and Zhang (2002) presented an approach to transform the grey fuzzy number into the interval number, and then utilized the ranking method of interval number to rank the order of alternatives. Basing on the grey fuzzy multiple attribute decision making in which both the fuzzy part and the grey part are real numbers. Jin and Lou (2004) used the decision making model which utilized the hamming distance to measure the alternatives and utilized the difference between the fuzzy positive ideal solution and the negative ideal solution to rank the order. In order to solve the grey fuzzy decision making, Luo and Liu (2004) developed the maximum entropy formulism to determine attribute weight and ranked the order of alternatives based on the linear combination of fuzzy information and grey information. Meng et al. (2007) proposed to present grey degree and fuzzy degree with the interval numbers, and then based on this, the mathematical model of interval valued grey fuzzy comprehensive evaluation was established. At last its application to the selection of the preferred project was given. In many real-life decision making problems, the linguistic variable is easier to express fuzzy information and closer to actual condition, the research on linguistic decision making has got rich achievements (Kacprzyk 1986; Delgado et al. 1992; Herrera et al. 1996; Herrera, Herrera-Viedma 2000; Merigó et al. 2010; Xu 2006, 2010; Zhou et al. 2008). Liu and Jin (2010) defined the concept of the interval grey linguistic variable which the fuzzy part and the grey part took the form of the linguistic variable and the interval number respectively, and studied the operation rules and developed the multiple attribute decision making method based on the interval grey linguistic variable. Liu and Zhang (2011) proposed the interval grey linguistic variables weighted geometric aggregation (IGLWGA) operator and the interval grey linguistic variables ordered weighted geometric aggregation (IGLOWGA) 
operator and the interval grey linguistic variables hybrid weighted geometric aggregation (IGLHWGA) operator, and then suggested a method for solving multiple attribute group decision making based on those operators.

All of the existing grey fuzzy multiple attribute decision making only considers the situation where all the elements in the grey fuzzy set are independent. However, in many practical situations, the elements in the grey fuzzy set are usually correlative. Therefore, we need to find some new ways to deal with these situations in which the decision data in question are correlative. The Choquet integral (Choquet 1953) is a very useful way of measuring the expected utility of an uncertain event, and can be utilized to depict the correlations of the decision data under consideration. Yager $(2003,2004 a)$ introduced the idea of order induced aggregation to the Choquet aggregation operator and defined an induced Choquet ordered averaging (C-OWA) operator, which allowed the ordering of the arguments to be based upon some other associated variables instead of ordering the arguments based on their values. Tan and Chen (2009) developed the induced Choquet ordered averaging (I-COA) operator and applied it to aggregate fuzzy preference relations in group decision making. $\mathrm{Xu}$ (2010) utilized the Choquet integral to propose the interval-valued intuitionistic fuzzy correlated averaging (IVIFCA) operator and the interval-valued intuitionistic fuzzy correlated geometric (IVIFCG) operator to aggregate interval-valued intuitionistic fuzzy information, and applied them to a practical decision-making problem involving the prioritization of information technology improvement projects.

Motivated by the correlation properties of the Choquet integral, in this paper, we propose some interval grey linguistic variables aggregation operators. The operators not only consider the importance of the elements, but also can reflect the correlations among the elements. To do so, the remainder of this paper is set out as follows. In the next section, we introduce some basic concepts related to grey fuzzy sets and some operational laws of interval grey linguistic variables. In Section 2 we have developed two interval grey linguistic correlated aggregation operators: interval grey linguistic correlated ordered arithmetic aggregation (IGLCOA) operator and interval grey linguistic correlated ordered geometric aggregation (IGLCOGA) operator. In Section 3, we have developed an approach to multiple attribute decision making based on IGLCOA operator and IGLCOGA operator with interval grey linguistic variables information. In Section 4, an illustrative example is pointed out. In final Section, we conclude the paper and give some remarks.

\section{Preliminaries}

In this section, we briefly review some basic concepts to be used throughout the paper.

Definition 1 (Chen 1994): Let $\tilde{A}(x)$ be the fuzzy subset in the space $X=\{x\}$, if the membership degree $\mu_{A}(x)$ of $x$ to $\tilde{A}(x)$ is the grey in the interval $[0,1]$, and its grey is $v_{A}(x)$, then $\tilde{A}(x)$ is called the grey fuzzy set in space $X$ (GF set, for short), denoted by $\tilde{A}(x)$, as follows: $\otimes$

$$
\underset{\otimes}{\tilde{A}}(x)=\left\{\left(x, \mu_{A}(x), v_{A}(x)\right) \mid x \in X\right\}
$$


The set pair mode is $\tilde{A}(x)=(\tilde{A}(x), A(x))$, where $\tilde{A}(x)=\left\{\left(x, \mu_{A}(x)\right) \mid x \in X\right\}$ is called the fuzzy part of $\underset{\otimes}{\tilde{A}}(x)$, and $\underset{\otimes}{A}(x)=\left\{\left(x, v_{A}(x)\right) \mid x \in X\right\}$ is called the grey part of $\tilde{\Theta}(x)$. So the grey fuzzy set is regarded as the generalization of the fuzzy set and the grey set.

Definition 2 (Liu, Jin 2010): Let $\underset{\otimes}{\tilde{A}}(x)=(\tilde{A}(x), \underset{\otimes}{A}(x))$ be the grey fuzzy number, if its fuzzy part is a linguistic variable $s_{\alpha} \in S$, where $S$ is a finite and totally ordered discrete term set, and its grey part $\underset{\otimes}{A}(x)$ is a closed interval $\left[g_{A}^{L}, g_{A}^{U}\right]$, then $\underset{\otimes}{\tilde{A}}(x)$ is called the interval grey linguistic variable.

The linguistic variable is easier to express fuzzy information, so it is reasonable to utilize the linguistic variables to represent the fuzzy part. While the grey part which indicated the amount of information obtained is described by the interval number, which can truly reflect the information the decision maker obtained. The larger the greyness of the grey part is, the less the obtained information is, and the lower the credibility of the obtained information is, that is, the lower the credibility of the obtained value is, the lower the value of the information is. When the greyness goes up to some extent, it means that the obtained information is useless. On the contrary, the smaller the greyness is, the more the obtained information is, the higher the credibility of the obtained information is, the more credible the obtained value is, the higher the value of the obtained information is.

Supposed that $\underset{\otimes}{\tilde{A}}(x)=\left(s_{\alpha},\left[g_{A}^{L}, g_{A}^{U}\right]\right), \underset{\otimes}{\tilde{B}}(x)=\left(s_{\beta},\left[g_{B}^{L}, g_{B}^{U}\right]\right)$ are two interval grey linguistic variables. The continuous ordered weighted averaging (C-OWA, for short) operator which is developed by Yager (2004b) can be usefully applied to aggregate the grey part, the greyness of the grey part would be transformed into a real number, and then the fuzzy part integrates with the grey part. That is to say, the size of the interval grey linguistic variables can get through comparing the size of $s_{\alpha} \times f_{\rho}\left(\left[\left(1-g_{A}^{L}\right),\left(1-g_{A}^{U}\right)\right]\right)$ and $s_{\beta} \times f_{\rho}\left(\left[\left(1-g_{B}^{L}\right),\left(1-g_{B}^{U}\right)\right]\right)$. Assume the ordering value $\left.Q \underset{\otimes}{\tilde{A}}(x))=s_{\alpha} \times f_{\rho}\left(\left[\left(1-g_{A}^{L}\right),\left(1-g_{A}^{U}\right)\right]\right), \underset{\otimes}{Q(\tilde{B}}(x)\right)=s_{\beta} \times f_{\rho}\left(\left[\left(1-g_{B}^{L}\right),\left(1-g_{B}^{U}\right)\right]\right)$, then $Q(\tilde{A}(x))=s_{\alpha-\alpha \times \int_{0}^{1} \frac{d \rho(y)}{d y}\left(g_{A}^{U}+y\left(g_{A}^{L}-g_{A}^{U}\right)\right) d y}, \underset{\otimes}{(\tilde{B}(x))=s_{\beta-\beta \times} \int_{0}^{1} \frac{d \rho(y)}{d y}\left(g_{B}^{U}+y\left(g_{B}^{L}-g_{B}^{U}\right)\right) d y}$, which can be obtained based on the continuous ordered weighted averaging (C-OWA) operator, such as $f_{p}([a, b])=\int_{0}^{1} \frac{d \rho(y)}{d y}(b-y(b-a)) d y$.

The operation rules of ranking are defined as follows:

1) If $Q(\underset{\otimes}{\tilde{A}}(x))>Q(\underset{\otimes}{\tilde{B}}(x))$, then we have $\underset{\otimes}{\tilde{A}}(x)>\underset{\otimes}{\tilde{B}}(x)$;

2) If $Q(\underset{\otimes}{\tilde{A}(x)})<Q(\underset{\otimes}{\tilde{B}}(x))$, then we have $\underset{\otimes}{\tilde{A}}(x)<\underset{\otimes}{\tilde{B}}(x)$;

3) If $Q(\underset{\otimes}{\tilde{A}}(x))=Q(\underset{\otimes}{\tilde{B}}(x))$ and $s_{\alpha} \geq s_{\beta}$, then we have $\underset{\otimes}{\tilde{A}}(x) \geq \underset{\otimes}{\tilde{B}}(x)$;

4) If $Q(\underset{\otimes}{\tilde{A}}(x))=Q(\underset{\otimes}{\tilde{B}}(x))$ and $s_{\alpha}<s_{\beta}$, then we have $\underset{\otimes}{\tilde{A}}(x)<\underset{\otimes}{\tilde{B}}(x)$. 
The function $\rho$ is denoted as basic unit-interval monotonic (BUM) functions. If $(\delta \geq 0)$ $\rho(y)=y^{\delta}$, then we have $Q(\underset{\otimes}{\tilde{A}}(x))=\alpha-\alpha \times \frac{\delta g_{A}^{L}+g_{A}^{U}}{\delta+1}$ and $\left.Q \underset{\otimes}{(\tilde{B}}(x)\right)=\beta-\beta \times \frac{\delta g_{B}^{L}+g_{B}^{U}}{\delta+1}$.

The operation rules of the interval grey linguistic variables are defined as follows:
1) $\underset{\otimes}{\tilde{A}}(x) \pm \underset{\otimes}{\tilde{B}}(x)=\left(s_{\alpha \pm \beta},\left[\left(1-\left(1-g_{A}^{L}\right) \times\left(1-g_{B}^{L}\right)\right),\left(1-\left(1-g_{A}^{U}\right) \times\left(1-g_{B}^{U}\right)\right)\right]\right)$;
2) $\underset{\otimes}{\tilde{A}}(x) \times \underset{\otimes}{\tilde{B}}(x)=\left(s_{\alpha \times \beta},\left[\left(1-\left(1-g_{A}^{L}\right) \times\left(1-g_{B}^{L}\right)\right),\left(1-\left(1-g_{A}^{U}\right) \times\left(1-g_{B}^{U}\right)\right)\right]\right)$;
3) $k \tilde{A}(x)=\left(s_{k \alpha},\left[g_{A}^{L}, g_{A}^{U}\right]\right)$;
4) $(\underset{\otimes}{\tilde{A}(x)})^{k}=\left(s_{\alpha^{k}},\left[g_{A}^{L}, g_{A}^{U}\right]\right)$.

Definition 3: An IGLOWA operator of dimension $n$ is a function IGLOWA: $\Omega^{n} \rightarrow \Omega$, which has associated a set of weights or weighting vector $w=\left(\omega_{1}, \omega_{2}, \cdots, \omega_{n}\right)$ with $\omega_{j} \in[0,1]$, $\sum_{j=1}^{n} \omega_{j}=1$, and is defined to aggregate a list of values $\left\{\underset{\otimes}{\tilde{A}}\left(x_{1}\right), \underset{\otimes}{\tilde{A}}\left(x_{2}\right), \cdots, \underset{\otimes}{\tilde{A}}\left(x_{n}\right)\right\}$ according to the following expression:

$$
\begin{aligned}
& \left.\operatorname{IGLOWA} \underset{\otimes}{\tilde{A}}\left(x_{1}\right), \underset{\otimes}{\tilde{A}}\left(x_{2}\right), \cdots, \underset{\otimes}{\tilde{A}}\left(x_{n}\right)\right)=\sum_{j=1}^{n} \omega_{j} \tilde{A}\left(x_{\tau(j)}\right)= \\
& \left(s_{\sum_{j=1}^{n} \omega_{j} \alpha_{\tau(j)}},\left[1-\prod_{j=1}^{n}\left(1-g_{\tau(j)}^{L}\right), 1-\prod_{j=1}^{n}\left(1-g_{\tau(j)}^{U}\right)\right],\right.
\end{aligned}
$$

where there is a permutation such that $\underset{\otimes}{\tilde{A}}\left(x_{\tau(1)}\right) \geq \underset{\otimes}{\tilde{A}}\left(x_{\tau(2)}\right) \geq \cdots \geq \underset{\otimes}{\tilde{A}}\left(x_{\tau(n)}\right)$, i.e., $\underset{\otimes}{\tilde{A}}\left(x_{\tau(j)}\right)$ the $j$ th largest value in the $\operatorname{set}\left\{\underset{\otimes}{\tilde{A}}\left(x_{1}\right), \underset{\otimes}{\tilde{A}}\left(x_{2}\right), \cdots, \underset{\otimes}{\tilde{A}}\left(x_{n}\right)\right\}$.

Definition 4 (Liu, Zhang 2010): An IGLOWG operator of dimension $n$ is a function IGLOWG: $\Omega^{n} \rightarrow \Omega$, which has associated a set of weights or weighting vector $w=\left(\omega_{1}, \omega_{2}, \cdots, \omega_{n}\right)$ with $\omega_{j} \in[0,1], \sum_{j=1}^{n} \omega_{j}=1$, and is defined to aggregate a list of values $\left\{\tilde{A}\left(x_{1}\right), \tilde{A}\left(x_{2}\right), \cdots, \tilde{A}\left(x_{n}\right)\right\}$ according to the following expression:

$$
\begin{aligned}
& \operatorname{IGLOWG}\left(\underset{\otimes}{\tilde{A}}\left(x_{1}\right), \underset{\otimes}{\tilde{A}}\left(x_{2}\right), \cdots, \underset{\otimes}{\tilde{A}}\left(x_{n}\right)\right)=\prod_{j=1}^{n}\left(\tilde{A}_{\otimes}^{\tilde{A}}\left(x_{\tau(j)}\right)\right)^{\omega_{j}}= \\
& \left(\prod_{j=1}^{n}\left(\alpha_{\tau(j)}\right)^{\omega_{j}},\left[\left(1-\prod_{j=1}^{n}\left(1-g_{\tau(j)}^{L}\right)\right),\left(1-\prod_{j=1}^{n}\left(1-g_{\tau(j)}^{U}\right)\right)\right]\right),
\end{aligned}
$$

where there is a permutation such that $\underset{\otimes}{\tilde{A}}\left(x_{\tau(1)}\right) \geq \underset{\otimes}{\tilde{A}}\left(x_{\tau(2)}\right) \geq \cdots \geq \underset{\otimes}{\tilde{A}}\left(x_{\tau(n)}\right)$, i.e., $\underset{\otimes}{\tilde{A}}\left(x_{\tau(j)}\right)$ the $j$ th largest value in the $\operatorname{set}\left\{\underset{\otimes}{\tilde{A}}\left(x_{1}\right), \underset{\otimes}{\tilde{A}}\left(x_{2}\right), \cdots, \underset{\otimes}{\tilde{A}}\left(x_{n}\right)\right\}$. 


\section{The interval grey linguistic correlated ordered arithmetic aggregation (IGLCOA) operator and the interval grey linguistic correlated ordered geometric aggregation (IGLCOGA) operator}

In multiple attribute decision making, the considered attributes usually have different levels of importance, and thus need to be assigned different weights. Some operators have been introduced to aggregate the interval grey linguistic variables together with independent weighted elements, but they only consider the addition of the importance of individual elements. However, in some practical situations, the elements in the interval grey linguistic variables have some correlations with each other, and thus it is necessary to consider this issue. For real decision making problems, there is always some degree of inter-dependent characteristics between attributes. Usually, there is an interaction among attributes of decision makers. However, this assumption is too strong to match decision behaviors in the real world. The independence axiom generally can not be satisfied. Thus, it is necessary to consider this issue.

Let $m\left(x_{i}\right)(i=1,2, \cdots, n)$ be the weight of the element $x_{i} \in X(i=1,2, \cdots, n)$, where $m$ is a fuzzy measure, defined as follows:

Definition 5 (Wang, Klir 1992): A fuzzy measure $m$ on the set $X$ is a set function $m: \theta(x) \rightarrow[0,1]$ satisfying the following axioms:

1) $m(\phi)=0, m(X)=1$;

2) $A \subseteq B$ implies $m(A) \leq m(B)$, for all $A, B \subseteq X$;

3) $m(A \cup B)=m(A)+m(B)+\rho m(A) m(B)$, for all $A, B \subseteq X$ and $A \cap B=\phi$, where $\rho \in(-1, \infty)$.

Especially, if $\rho=0$, then the condition (3) reduces to the axiom of additive measure:

$$
m(A \cup B)=m(A)+m(B), \text { for all } A, B \subseteq X \text { and } A \cap B=\phi .
$$

If all the elements in $X$ are independent, and we have

$$
m(A)=\sum_{x_{i} \in A} m\left(\left\{x_{i}\right\}\right), \text { for all } A \subseteq X .
$$

Based on Definition 5, in what follows we use the well-known Choquet integral (Choquet 1953) to develop an operator for aggregating the interval grey linguistic variables with correlative weights:

Definition 6: Let $m$ be a fuzzy measure on $X$, and $\underset{\otimes}{A}\left(x_{j}\right)=\left(s_{\alpha_{j}},\left[g_{j}^{L}, g_{j}^{U}\right]\right)(j=1,2, \cdots, n)$ be $n$ interval grey linguistic variables, then we call

$$
\begin{aligned}
& \left(C_{1}\right) \int_{\otimes} \underset{\Theta}{\tilde{A}}(x) d m=\operatorname{IGLCOA}\left(\underset{\otimes}{\tilde{A}}\left(x_{1}\right), \underset{\otimes}{\tilde{A}}\left(x_{2}\right), \cdots, \underset{\otimes}{\tilde{A}}\left(x_{n}\right)\right)= \\
& \underset{j=1}{\oplus}\left(m\left(B\left(x_{\tau(j)}\right)\right)-m\left(B\left(x_{\tau(j-1)}\right)\right)\right) \underset{\otimes}{\tilde{A}}\left(x_{\tau(j)}\right),
\end{aligned}
$$

the interval grey linguistic correlated ordered arithmetic averaging (IGLCOA) operator, where $\left(C_{1}\right) \int_{\otimes} \underset{\otimes}{\tilde{A}} d m$ denotes the Choquet integral, $(\tau(1), \tau(2), \cdots, \tau(n))$ is a permutation of $(1,2, \cdots, n)$ 
such that $\underset{\otimes}{\tilde{A}}\left(x_{\tau(1)}\right) \geq \underset{\Theta}{\tilde{A}}\left(x_{\tau(2)}\right) \geq \cdots \geq \underset{\Theta}{\tilde{A}}\left(x_{\tau(n)}\right), B\left(x_{\tau(j)}\right)=\left\{x_{\tau(k)} \mid k \leq j, k \geq 1\right\}$ and $B\left(x_{\tau(0)}\right)=\phi$. Whose aggregated value is also an interval grey linguistic variable (IGLV, for short).

Below, we discuss two special cases of the IGLCOA operator:

1) If $\rho=0$, then $m(B \cup C)=m(B)+m(C)$ and $m\left(\left\{x_{\tau(j)}\right\}\right)=m\left(B\left(x_{\tau(j)}\right)\right)-m\left(B\left(x_{\tau(j-1)}\right)\right)$, $j=1,2, \cdots, n$. In this case, the IGLCOA operator (6) reduces to the interval grey linguistic weighted arithmetic averaging (IGLWAA) operator:

$$
\begin{aligned}
& \operatorname{IGLWAA}\left(\underset{\otimes}{\tilde{A}}\left(x_{1}\right), \underset{\otimes}{\tilde{A}}\left(x_{2}\right), \cdots, \underset{\otimes}{\tilde{A}}\left(x_{n}\right)\right)=\bigoplus_{j=1}^{n} m\left(\left\{x_{j}\right\}\right) \underset{\otimes}{\tilde{A}}\left(x_{j}\right)= \\
& \left(s_{j=1}^{n} m\left(\left\{x_{j}\right\}\right) \alpha_{j},\left[1-\prod_{j=1}^{n}\left(1-g_{j}^{L}\right), 1-\prod_{j=1}^{n}\left(1-g_{j}^{U}\right)\right] .\right.
\end{aligned}
$$

In particular, if $m\left(\left\{x_{j}\right\}\right)=\frac{1}{n}$ for all $j=1,2, \cdots, n$, then the IGLWAA operator (7) reduces to the interval grey linguistic arithmetic averaging (IGLAA) operator:

$$
\begin{aligned}
& \operatorname{IGLAA}\left(\underset{\Theta}{\tilde{A}}\left(x_{1}\right), \underset{\otimes}{\tilde{A}}\left(x_{2}\right), \cdots, \underset{\otimes}{\tilde{A}}\left(x_{n}\right)\right)= \\
& \left.\left\{s_{\sum_{j=1}^{n} \frac{\alpha_{j}}{n},}, 1-\prod_{j=1}^{n}\left(1-g_{\tau(j)}^{L}\right), 1-\prod_{j=1}^{n}\left(1-g_{\tau(j)}^{U}\right)\right]\right\} .
\end{aligned}
$$

2) If $m(B)=\sum_{j=1}^{B} \omega_{j}$ for all $B \subseteq X$, where $|B|$ is the number of the elements in the set $B$, then $\omega_{j}=m\left(B\left(x_{\tau(j)}\right)\right)-m\left(B\left(x_{\tau(j-1)}\right)\right), j=1,2, \cdots, n$, where $\omega=\left(\omega_{1}, \omega_{2}, \cdots, \omega_{n}\right)^{T}, \omega_{j} \geq 0$, $j=1,2, \cdots, n$, and $\sum_{j=1}^{n} \omega_{j}=1$. In this case, the IGLCOA operator (6) reduces to the interval grey linguistic ordered weighted arithmetic averaging (IGLOWA) operator:

$$
\begin{aligned}
& \operatorname{IGLOWA}\left(\underset{\otimes}{\tilde{A}}\left(x_{1}\right), \underset{\otimes}{\tilde{A}}\left(x_{2}\right), \cdots, \underset{\otimes}{\tilde{A}}\left(x_{n}\right)\right)=\bigoplus_{j=1}^{n} \omega_{j} \underset{\otimes}{\tilde{A}}\left(x_{\tau(j)}\right)= \\
& \left(s_{\sum_{j=1}^{n} \omega_{j} \alpha_{\tau(j)}},\left[1-\prod_{j=1}^{n}\left(1-g_{\tau(j)}^{L}\right), 1-\prod_{j=1}^{n}\left(1-g_{\tau(j)}^{U}\right)\right] .\right.
\end{aligned}
$$

In particular, if $m(B)=\frac{|B|}{n}$ for all $B \subseteq X$, then both the IGLCOA operator (6) and the IGLOWA operator (9) reduce to the IGLAA operator. 
Definition 7: Let $m$ be a fuzzy measure on $X$, and $\underset{\otimes}{\tilde{A}}\left(x_{j}\right)=\left(s_{\alpha_{j}},\left[g_{j}^{L}, g_{j}^{U}\right]\right)(j=1,2, \cdots, n)$ be $n$ interval grey linguistic variables, then we call

$$
\begin{aligned}
& \left(C_{2}\right) \int_{\otimes}^{\tilde{A}}(x) d m=\operatorname{IGLCOGA}\left(\underset{\otimes}{\tilde{A}}\left(x_{1}\right), \underset{\otimes}{\tilde{A}}\left(x_{2}\right), \cdots, \underset{\otimes}{\tilde{A}}\left(x_{n}\right)\right)= \\
& \underset{j=1}{\otimes}\left(\underset{\Theta}{\tilde{A}}\left(x_{\tau(j)}\right)\right)^{m\left(B\left(x_{\tau(j)}\right)\right)-m\left(B\left(x_{\tau(j-1)}\right)\right)},
\end{aligned}
$$

the interval grey linguistic correlated ordered geometric averaging (IGLCOGA) operator, where $\left(C_{2}\right) \int_{\otimes} \underset{\Theta}{\tilde{A}}(x) d m$ denotes the Choquet integral, $(\tau(1), \tau(2), \cdots, \tau(n))$ is a permutation of $(1,2, \cdots, n)$ such that $\underset{\otimes}{\tilde{A}}\left(x_{\tau(1)}\right) \geq \underset{\Theta}{\tilde{A}}\left(x_{\tau(2)}\right) \geq \cdots \geq \underset{\otimes}{\tilde{A}}\left(x_{\tau(n)}\right), B\left(x_{\tau(j)}\right)=\left\{x_{\tau(k)} \mid k \leq j, k \geq 1\right\}$ and $B\left(x_{\tau(0)}\right)=\phi$.

The IGLCOGA operator (10) can be transformed into the following form by induction on $n$ :

$$
\begin{aligned}
& \left(C_{2}\right) \int_{\otimes} \underset{\Theta}{\tilde{A}}(x) d m=\operatorname{IGLCOGA}\left(\underset{\otimes}{\tilde{A}}\left(x_{1}\right), \underset{\otimes}{\tilde{A}}\left(x_{2}\right), \cdots, \underset{\otimes}{\tilde{A}}\left(x_{n}\right)\right)= \\
& \left(\prod_{j=1}^{s_{n}^{n}\left(\alpha_{\tau(j)}\right)}{ }^{m\left(B_{\tau(j)}\right)-m\left(B_{\tau(j-1)}\right)},\left[1-\prod_{j=1}^{n}\left(1-g_{\tau(j)}^{L}\right), 1-\prod_{j=1}^{n}\left(1-g_{\tau(j)}^{U}\right)\right] .\right.
\end{aligned}
$$

Whose aggregated value is also an interval grey linguistic variable (IGLV, for short).

Especially, if $m\left(\left\{x_{\tau(j)}\right\}\right)=m\left(B\left(x_{\tau(j)}\right)\right)-m\left(B\left(x_{\tau(j-1)}\right)\right), j=1,2, \cdots, n$, then IGLCOGA operator reduces to IGLWGA operator. If $m(B)=\sum_{j=1}^{|B|} \omega_{j}$, for all $B \subseteq X$, where $|B|$ is the number of the elements in the set $B$, then $\omega_{j}=m\left(B\left(x_{\tau(j)}\right)\right)-m\left(B\left(x_{\tau(j-1)}\right)\right), j=1,2, \cdots, n$, where $\omega=\left(\omega_{1}, \omega_{2}, \cdots, \omega_{n}\right)^{T}, \omega_{j} \geq 0, j=1,2, \cdots, n$, and $\sum_{j=1}^{n} \omega_{j}=1$. Then, IGLCOGA operator reduces to IGLOWGA operator.

\section{The multiple attribute group decision making method based on the interval grey linguistic correlated ordered aggregation (IGLCOA) operator and the interval grey linguistic correlated ordered geometric aggregation (IGLCOGA) operator}

In this section, we shall develop an approach to multiple attribute decision making with correlative weights and interval grey linguistic variables information as follows.

Let $A=\left\{a_{1}, a_{2}, \cdots, a_{m}\right\}$ be a discrete set of alternatives, $X=\left\{x_{1}, x_{2}, \cdots, x_{n}\right\}$ be the set of attributes and $m$ be a fuzzy measure on $X$, where $0 \leq m\left(\left\{x_{1}, \cdots, x_{j}\right\}\right) \leq 1, m(\{X\})=1$ and 

$m(\{\phi\})=0$. Supposed that $\underset{\otimes}{\tilde{A}}\left(x_{j}\right)=\left(s_{\alpha_{i j}},\left[g_{i j}^{L}, g_{i j}^{U}\right]\right)$ is the attribute value in the attribute set $x_{j}$ with respect to the alternative $a_{i}$ which given by experts and $R=\left(\underset{\otimes_{a_{i}}}{\tilde{A}}\left(x_{j}\right)\right)_{m \times n}$ is the decision making matrix.

In the following, we apply the IGLCOA operator and the IGLCOGA operator to multiple attribute decision making with correlative weights, which attribute values are given in terms of interval grey linguistic variables information.

Step 1 . We rearrange the interval grey linguistic variables corresponding to each project in descending order by using the method presented in Section 1.

$$
Q(\tilde{A}(x))=\alpha-\alpha \times \int_{0}^{1} \frac{d \rho(y)}{d y}\left(g_{A}^{U}+y\left(g_{A}^{L}-g_{A}^{U}\right)\right) d y .
$$

Step 2. With the IGLCOA operator (6) or the IGLCOGA operator (10), we calculate the overall evaluation information corresponding to each project.

$$
\begin{aligned}
& z_{i}=\operatorname{IGLCOA}\left(\underset{\otimes_{a_{i}}}{\tilde{A}}\left(x_{1}\right), \underset{\otimes_{a_{i}}}{\tilde{A}}\left(x_{2}\right), \cdots, \underset{\otimes_{a_{i}}}{\tilde{A}}\left(x_{n}\right)\right)= \\
& \oplus_{j=1}^{n}\left(m\left(B\left(x_{\tau(j)}\right)\right)-m\left(B\left(x_{\tau(j-1)}\right)\right)\right) \underset{\otimes_{a_{i}}}{\tilde{A}}\left(x_{j}\right), i=1,2, \cdots, m ; \\
& z_{i}=\operatorname{IGLCOGA}\left(\underset{\otimes a_{i}}{\tilde{A}}\left(x_{1}\right), \underset{\otimes a_{i}}{\tilde{A}}\left(x_{2}\right), \cdots, \underset{\otimes a_{i}}{\tilde{A}}\left(x_{n}\right)\right)= \\
& \bigotimes_{j=1}^{n}\left(\tilde{A}_{\otimes_{a_{i}}}\left(x_{j}\right)\right)^{m\left(B\left(x_{\tau(j)}\right)\right)-m\left(B\left(x_{\tau(j-1)}\right)\right)}, i=1,2, \cdots, m .
\end{aligned}
$$

Step 3. We rank the above interval grey linguistic variables by using the method presented in Section 1. The ranking of the alternatives can be gained and the best one can be find out.

\section{Practical example}

A practical use of the proposed approach involves evaluating the technological innovation ability of the six enterprises $\left\{a_{1}, a_{2}, a_{3}, a_{4}, a_{5}, a_{6}\right\}$, the attributes is shown as follows: the ability of innovative resources input $\left(x_{1}\right)$; the ability of innovation tendency $\left(x_{2}\right)$; the ability of innovation management $\left(x_{3}\right)$. Based on the three attributes, the attribute values given by the experts take the form of the interval grey linguistic variables, shown in Tables 1 . Let $S=\left(s_{0}, s_{1}, s_{2}, s_{3}, s_{4}, s_{5}, s_{6}\right)$ be the linguistic label and the attribute weight be correlative, which is given as follows (Herrera, Herrera-Viedma 2000): $S=\left(s_{0}, s_{1}, s_{2}, s_{3}, s_{4}, s_{5}, s_{6}\right)=$ \{very poor, poor, slightly poor, fair, slightly good, good, very good\}. The problem is ranking the six enterprises based on their technological innovation ability. The evaluation steps used in this paper are proposed as follows: 
Table 1. The attribute values of each attribute with respect to six enterprises given by experts

\begin{tabular}{cccc}
\hline Enterprises & Attribute $\left(x_{1}\right)$ & Attribute $\left(x_{2}\right)$ & Attribute $\left(x_{3}\right)$ \\
\hline$a_{1}$ & $\left(s_{5},[0.2,0.3]\right)$ & $\left(s_{2},[0.4,0.4]\right)$ & $\left(s_{5},[0.5,0.5]\right)$ \\
$a_{2}$ & $\left(s_{4},[0.4,0.4]\right)$ & $\left(s_{5},[0.4,0.5]\right)$ & $\left(s_{3},[0.1,0.2]\right)$ \\
$a_{3}$ & $\left(s_{3},[0.2,0.3]\right)$ & $\left(s_{4},[0.2,0.3]\right)$ & $\left(s_{4},[0.3,0.3]\right)$ \\
$a_{4}$ & $\left(s_{6},[0.5,0.6]\right)$ & $\left(s_{2},[0.2,0.2]\right)$ & $\left(s_{3},[0.2,0.4]\right)$ \\
$a_{5}$ & $\left(s_{4},[0.1,0.3]\right)$ & $\left(s_{3},[0.2,0.3]\right)$ & $\left(s_{3},[0.2,0.2]\right)$ \\
$a_{6}$ & $\left(s_{5},[0.4,0.5]\right)$ & $\left(s_{3},[0.3,0.4]\right)$ & $\left(s_{4},[0.2,0.4]\right)$ \\
\hline
\end{tabular}

The experts evaluate the enterprises $a_{i}(i=1,2,3,4,5,6)$ in relation to the factors $x_{j}(j=1,2,3)$, and give more importance to $x_{1}$ and $x_{2}$ than to $x_{3}$, but, on the other hand, the experts give some advantage to the enterprises that are good both in $x_{3}$ and in either of $x_{1}$ and $x_{2}$. Let

$$
\begin{aligned}
& m(\phi)=0, m(X)=m\left(\left\{x_{1}, x_{2}, x_{3}\right\}\right)=1, m\left(\left\{x_{1}\right\}\right)=m\left(\left\{x_{2}\right\}\right)=0.4, \\
& m\left(\left\{x_{3}\right\}\right)=0.3, m\left(\left\{x_{1}, x_{2}\right\}\right)=0.6, m\left(\left\{x_{1}, x_{3}\right\}\right)=m\left(\left\{x_{2}, x_{3}\right\}\right)=0.8
\end{aligned}
$$

Step 1. We rearrange the interval grey linguistic variables corresponding to each project in descending order, by using the method presented in Section 1. Supposed that the basic unit-interval monotonic (BUM) function is $\rho(y)=y^{2}$, and then the ordering value can be get.

$$
\begin{aligned}
& Q\left(\underset{\otimes_{a_{1}}}{\tilde{\tilde{A}}}\left(x_{1}\right)\right)=s_{3.833} ; Q\left(\underset{\otimes_{a_{1}}}{\tilde{A}}\left(x_{2}\right)\right)=s_{1.200} ; Q\left(\underset{\otimes_{a_{1}}}{\tilde{A}}\left(x_{3}\right)\right)=s_{2.500} ; \\
& Q\left(\underset{\otimes_{a 2}}{\tilde{A}}\left(x_{1}\right)\right)=s_{2.400} ; Q\left(\underset{\otimes_{a 2}}{\tilde{A}}\left(x_{2}\right)\right)=s_{2.833} ; Q\left(\underset{\otimes_{a 2}}{\tilde{A}}\left(x_{3}\right)\right)=s_{2.600} ; \\
& Q\left(\underset{\otimes_{a_{3}}}{\tilde{A}}\left(x_{1}\right)\right)=s_{2.300} ; Q\left(\underset{\otimes_{a_{3}}}{\tilde{A}}\left(x_{2}\right)\right)=s_{3.067} ; Q\left(\underset{\otimes_{a_{3}}}{\tilde{\tilde{A}}}\left(x_{3}\right)\right)=s_{2.800} ; \\
& Q\left(\underset{\otimes_{a_{4}}}{\tilde{A}}\left(x_{1}\right)\right)=s_{2.800} ; Q\left(\underset{\otimes_{a_{4}}}{\tilde{A}}\left(x_{2}\right)\right)=s_{1.600} ; Q\left(\underset{\otimes_{a_{4}}}{\tilde{A}}\left(x_{3}\right)\right)=s_{2.200} ; \\
& Q\left(\underset{\otimes_{a_{5}}}{\tilde{A}}\left(x_{1}\right)\right)=s_{3.333} ; Q\left(\underset{\otimes_{a_{5}}}{\tilde{\tilde{A}}}\left(x_{2}\right)\right)=s_{2.300} ; Q\left(\underset{\otimes_{a_{5}}}{\tilde{A}}\left(x_{3}\right)\right)=s_{2.400} ; \\
& Q\left(\underset{\otimes_{a_{6}}}{\tilde{A}}\left(x_{1}\right)\right)=s_{2.833} ; Q\left(\underset{\otimes_{a_{6}}}{\tilde{A}}\left(x_{2}\right)\right)=s_{2.000} ; Q\left(\underset{\otimes_{a_{6}}}{\tilde{A}}\left(x_{3}\right)\right)=s_{2.933} \text {. }
\end{aligned}
$$

Step 2. With the IGLCOA operator (13), we calculate the overall evaluation information corresponding to each project. Where $Q\left(\underset{\otimes_{a_{1}}}{\tilde{A}}\left(x_{1}\right)\right) \geq Q\left(\underset{\otimes_{a_{1}}}{\tilde{A}}\left(x_{3}\right)\right) \geq Q\left(\underset{\otimes_{a_{1}}}{\tilde{A}}\left(x_{2}\right)\right)$, then $\underset{\otimes}{\tilde{A}}\left(x_{\tau(1)}\right)=\underset{\otimes_{a_{1}}}{\tilde{A}}\left(x_{1}\right), \underset{\otimes_{a_{1}}}{\tilde{A}}\left(x_{\tau(2)}\right)=\underset{\otimes_{a_{1}}}{\tilde{A}}\left(x_{3}\right), \underset{\otimes_{a_{1}}}{\tilde{A}}\left(x_{\tau(3)}\right)=\underset{\otimes_{a_{1}}}{\tilde{A}}\left(x_{2}\right)$. We have $m\left(B\left(x_{\tau(1)}\right)\right)=m\left(\left\{x_{1}\right\}\right)=0.4$ 


$$
\begin{aligned}
& m\left(B\left(x_{\tau(2)}\right)\right)=m\left(\left\{x_{1}, x_{3}\right\}\right)=0.8, m\left(B\left(x_{\tau(3)}\right)\right)=m\left(\left\{x_{1}, x_{2}, x_{3}\right\}\right)=1 . \\
& z_{1}=\operatorname{IGLCOA}\left(\underset{\otimes_{a_{1}}}{\tilde{A}}\left(x_{1}\right), \underset{\otimes_{a_{1}}}{\tilde{A}}\left(x_{2}\right), \underset{\otimes_{a_{1}}}{\tilde{A}}\left(x_{3}\right)\right)= \\
& \left.\underset{j=1}{\oplus}\left(m\left(B\left(x_{\tau(j)}\right)\right)-m\left(B\left(x_{\tau(j-1)}\right)\right)\right)\right)_{\otimes_{a_{1}}}^{\tilde{A}}\left(x_{j}\right)=\left(s_{4.4},[0.76,0.79]\right) .
\end{aligned}
$$

We can also use the same method to obtain the other overall preference values $z_{i}$ of the alternatives $a_{i}(i=1,2, \cdots, 6)$.

$$
\begin{aligned}
& z_{1}=\left(s_{4.4},[0.76,0.79]\right) ; z_{2}=\left(s_{4},[0.676,0.760]\right) ; z_{3}=\left(s_{3.8},[0.552,0.657]\right) ; \\
& z_{4}=\left(s_{4},[0.68,0.808]\right) ; z_{5}=\left(s_{3.4},[0.424,0.608]\right) ; z_{6}=\left(s_{4.3},[0.664,0.82]\right) .
\end{aligned}
$$

Step 3. We rank the above interval grey linguistic variables by using the method presented in Section 1:

$$
\begin{aligned}
& Q\left(z_{1}\right)=s_{1.0120} ; Q\left(z_{2}\right)=s_{1.1840} ; Q\left(z_{3}\right)=s_{1.5694} \\
& Q\left(z_{4}\right)=s_{1.1093} ; Q\left(z_{5}\right)=s_{1.7499} ; Q\left(z_{6}\right)=s_{1.2212}
\end{aligned}
$$

The ranking of the alternatives can be gained: $z_{5} \succ z_{3} \succ z_{6} \succ z_{2} \succ z_{4} \succ z_{1}, a_{5}$ is the best one.

If we use the IGLCOGA operator (14) to calculate the overall evaluation information corresponding to each enterprise, then

$$
\begin{aligned}
& z_{1}=\operatorname{IGLCOGA}\left(\underset{\otimes_{a_{1}}}{\tilde{A}}\left(x_{1}\right), \underset{\otimes_{a_{1}}}{\tilde{A}}\left(x_{2}\right), \underset{\otimes_{a_{1}}}{\tilde{A}}\left(x_{3}\right)\right)=\left(s_{4.163},[0.76,0.79]\right) ; \\
& z_{2}=\operatorname{IGLCOGA}\left(\underset{\otimes_{a_{2}}}{\tilde{\tilde{A}}}\left(x_{1}\right), \underset{\otimes_{a_{2}}}{\tilde{A}}\left(x_{2}\right), \underset{\otimes_{a_{2}}}{\tilde{A}}\left(x_{3}\right)\right)=\left(s_{3.898},[0.676,0.76]\right) ; \\
& z_{3}=\operatorname{IGLCOGA}\left(\underset{\otimes_{a_{3}}}{\tilde{A}}\left(x_{1}\right), \underset{\otimes_{a_{3}}}{\tilde{A}}\left(x_{2}\right), \underset{\otimes_{a_{3}}}{\tilde{A}}\left(x_{3}\right)\right)=\left(s_{3.776},[0.552,0.657]\right) ; \\
& z_{4}=\operatorname{IGLCOGA}\left(\underset{\otimes_{a_{4}}}{\tilde{A}}\left(x_{1}\right), \underset{\otimes_{a_{4}}}{\tilde{A}}\left(x_{2}\right), \underset{\otimes_{a_{4}}}{\tilde{A}}\left(x_{3}\right)\right)=\left(s_{3.650},[0.68,0.808]\right) ; \\
& z_{5}=\operatorname{IGLCOGA}\left(\underset{\otimes_{a_{5}}}{\tilde{A}}\left(x_{1}\right), \underset{\otimes_{a_{5}}}{\tilde{A}}\left(x_{2}\right), \underset{\otimes_{a_{5}}}{\tilde{A}}\left(x_{3}\right)\right)=\left(s_{3.366},[0.424,0.608]\right) ; \\
& z_{6}=\operatorname{IGLCOGA}\left(\underset{\otimes_{a_{6}}}{\tilde{\tilde{A}}}\left(x_{1}\right), \underset{\otimes_{a_{6}}}{\tilde{A}}\left(x_{2}\right), \underset{\otimes_{a_{6}}}{\tilde{A}}\left(x_{3}\right)\right)=\left(s_{4.122},[0.664,0.82]\right) .
\end{aligned}
$$

And we rank those interval grey linguistic variables by using the method presented in Section 1:

$$
\begin{aligned}
& Q\left(z_{1}\right)=s_{0.9574} ; Q\left(z_{2}\right)=s_{1.1538} ; Q\left(z_{3}\right)=s_{1.5596} \\
& Q\left(z_{4}\right)=s_{1.0123} ; Q\left(z_{5}\right)=s_{1.7323} ; Q\left(z_{6}\right)=s_{1.1991}
\end{aligned}
$$


The ranking of the alternatives can be gained: $z_{5} \succ z_{3} \succ z_{6} \succ z_{2} \succ z_{4} \succ z_{1}, a_{5}$ is the best one.

Which indicates that the IGLCOA operator and the IGLCOGA operator produce the same ranking results, the best project is $a_{5}$ in both methods.

According the comparison method in Section 2, we can get the order of alternatives in Table 2. As we can see, the order of alternatives is slightly different as $\delta$ increases, which results in the indeterminacy of the final decision, because the different parameter $\delta$ would cause the different weights of numbers in the grey part. It is very reasonable that $\delta$ can be considered as the decision makers' risk preference similar to the parameter defined by Yager (2004a, b). As a result, the investor can properly select the desirable alternative in accordance with his/her interest and the actual needs.

Table 2. Ordering of the alternatives

\begin{tabular}{ccc}
\hline & Ordering & Ordering \\
& (Using the IGLCOA operator) & (Using IGLCOGA operator) \\
\hline$\delta=0.1$ & $\mathrm{z}_{5} \succ \mathrm{z}_{3} \succ \mathrm{z}_{2} \succ \mathrm{z}_{1} \succ \mathrm{z}_{6} \succ \mathrm{z}_{4}$ & $\mathrm{z}_{5} \succ \mathrm{z}_{3} \succ \mathrm{z}_{2} \succ \mathrm{z}_{1} \succ \mathrm{z}_{4} \succ \mathrm{z}_{6}$ \\
$\delta=1$ & $\mathrm{z}_{5} \succ \mathrm{z}_{3} \succ \mathrm{z}_{2} \succ \mathrm{z}_{6} \succ \mathrm{z}_{1} \succ \mathrm{z}_{4}$ & $\mathrm{z}_{5} \succ \mathrm{z}_{3} \succ \mathrm{z}_{2} \succ \mathrm{z}_{6} \succ \mathrm{z}_{1} \succ \mathrm{z}_{4}$ \\
$\delta=5$ & $\mathrm{z}_{5} \succ \mathrm{z}_{3} \succ \mathrm{z}_{2} \succ \mathrm{z}_{6} \succ \mathrm{z}_{4} \succ \mathrm{z}_{1}$ & $\mathrm{z}_{5} \succ \mathrm{z}_{3} \succ \mathrm{z}_{2} \succ \mathrm{z}_{6} \succ \mathrm{z}_{4} \succ \mathrm{z}_{1}$ \\
$\delta=10$ & $\mathrm{z}_{5} \succ \mathrm{z}_{3} \succ \mathrm{z}_{2} \succ \mathrm{z}_{6} \succ \mathrm{z}_{4} \succ \mathrm{z}_{1}$ & $\mathrm{z}_{5} \succ \mathrm{z}_{3} \succ \mathrm{z}_{2} \succ \mathrm{z}_{6} \succ \mathrm{z}_{4} \succ \mathrm{z}_{1}$ \\
$\delta=50$ & $\mathrm{z}_{5} \succ \mathrm{z}_{3} \succ \mathrm{z}_{2} \succ \mathrm{z}_{6} \succ \mathrm{z}_{4} \succ \mathrm{z}_{1}$ & $\mathrm{z}_{5} \succ \mathrm{z}_{3} \succ \mathrm{z}_{2} \succ \mathrm{z}_{6} \succ \mathrm{z}_{4} \succ \mathrm{z}_{1}$ \\
\hline
\end{tabular}

\section{Conclusions}

In this paper, we have developed the interval grey linguistic variables ordered weighted aggregation (IGLOWA) operator, and then use the Choquet integral to propose the interval grey linguistic correlated ordered arithmetic aggregation (IGLCOA) operator and the interval grey linguistic correlated ordered geometric aggregation (IGLCOGA) operator, which are used to discuss the correlative interval grey linguistic variables. Furthermore, we also analyze the relation between it and some known operators and have developed an approach to multiple attribute decision making with correlative weights which the attribute values are given in terms of interval grey linguistic variables information based on the interval grey linguistic correlated ordered arithmetic aggregation (IGLCOA) operator and the interval grey linguistic correlated ordered geometric aggregation (IGLCOGA) operator. Finally an illustrative example has been given to show the developed method. The applications of the operator in many actual fields, such as decision making, pattern recognition and clustering analysis, are open questions for future research.

\section{Acknowledgements}

The research was supported by National Natural Science Foundation of China (No.90924012, No.71090402, No.71103149); Program for New Century Excellent Talents in University (No. NCET-10-0706); Specialized Research Fund for the Doctoral Program of Higher Education (No.20090184110029); Humanities and Social Science Research Foundation of 
the Ministry of Education (No.08JC630067); Sichuan Youth Science and Technology Foundation (No.09ZQ026-021); Fund for Cultivating Academic and Technological Leaders in Sichuan Province (No.2011-441); Fundamental Research Funds for the Central Universities (No. SWJTU11CX152).

\section{References}

Baležentis, T.; Zeng, S. 2013. Group multi-criteria decision making based upon interval-valued fuzzy numbers: an extension of the MULTIMOORA method, Expert Systems With Applications 40(2): 543-550. http://dx.doi.org/10.1016/j.eswa.2012.07.066

Bu, G. Z.; Zhang, Y. W. 2002. Grey fuzzy comprehensive evaluation based on the theory of grey fuzzy relation, Systems Engineering-Theory and Practice 22(4): 141-144.

Chen, D. W. 1994. Grey fuzzy set introduction. Harbin: Heilongjiang Science and Technology Press.

Choquet, G. 1953. Theory of capacities, Annales de l'institut Fourier 5(1): 131-295. http://dx.doi.org/10.5802/aif.53

Delgado, M.; Verdegay, J.; Vila, M. 1992. Linguistic decision making models, International Journal of Intelligent Systems 7(5): 479-492. http://dx.doi.org/10.1002/int.4550070507

Deng, J. L. 1982. Control problems of grey systems, Systems and Control Letters 1(5): 288-294. http://dx.doi.org/10.1016/S0167-6911(82)80025-X

Herrera, F.; Herrera-Viedma, E. 2000. Linguistic decision analysis: steps for solving decision problems under linguistic information, Fuzzy Sets and Systems 115(1): 67-82. http://dx.doi.org/10.1016/S0165-0114(99)00024-X

Herrera, F.; Herrera-Viedma, E.; Verdegay, J. 1996. Direct approach processes in group decision making using linguistic OWA operators, Fuzzy Sets and Systems 79(2): 175-190. http://dx.doi.org/10.1016/0165-0114(95)00162-X

Jin, N.; Lou, S. C. 2004. A grey fuzzy multi-attribute decision making method, Fire Control and Command Control 29(4): 26-28.

Kacprzyk, J. 1986. Group decision making with a fuzzy linguistic majority, Fuzzy Sets and Systems 18(2): 105-118. http://dx.doi.org/10.1016/0165-0114(86)90014-X

Li, P. 2012. Intuitionistic fuzzy decision-making methods based on grey incidence analysis and DS theory of evidence, Grey Systems: Theory and Application 2(1): 54-62. http://dx.doi.org/10.1108/20439371211197677

Liu, P. D.; Jin, F. 2010. The multi-attribute group decision making method based on the interval grey linguistic variables, African Journal of Business Management 4(17): 3708-3715.

Liu, P. D.; Zhang, X. 2011. The multi-attribute group decision making method based on the interval grey linguistic variables weighted geometric aggregation operator, Control and Decision 26(5): 743-747.

Luo, D.; Liu, S. F. 2004. Analytic method to a kind of grey fuzzy decision making based on entropy, Engineering Science 6(10): 48-51.

Meng, K.; Li, Y. W.; Wang, C. J. 2007. Interval-value grey fuzzy comprehensive evaluation based on the preference of the risk and its application, Fire Control Command Control 32(4): 109-111.

Merigó, J. M. 2010. Fuzzy decision making with immediate probabilities, Computers and Industrial Engineering 58(4): 651-657. http://dx.doi.org/10.1016/j.cie.2010.01.007

Merigó, J. M.; Gil-Lafuente, A. M. 2011. Fuzzy induced generalized aggregation operators and its application in multi-person decision making, Expert Systems with Applications 38(8): 9761-9772.

http://dx.doi.org/10.1016/j.eswa.2011.02.023 
Merigó, J. M.; Casanovas, M.; Martínez, L. 2010. Linguistic aggregation operators for linguistic decision making based on the Dempster-Shafer theory of evidence, International Journal of Uncertainty, Fuzziness and Knowledge-Based Systems 18(3): 287-304. http://dx.doi.org/10.1142/S0218488510006544

Salmeron, J. L.; Gutierrez, E. 2012. Fuzzy grey cognitive maps in reliability engineering, Applied Soft Computing 12(12): 3818-3824. http://dx.doi.org/10.1016/j.asoc.2012.02.003

Tan, C. Q.; Chen, X. H. 2009. Induced Choquet ordered averaging operator and its application to group decision making, International Journal of Intelligent Systems 25(1): 59-82. http://dx.doi.org/10.1002/int.20388

Wang, Q. Y., et al. 1996. Grey fuzzy mathematical foundation. Wuhan: Huazhong University of Science and Technology Press.

Wang, Z. Y.; Klir, G. J. 1992. Fuzzy measure theory. New York: Plenum Publishing Corporation.

Wei, G. W.; Wang, H. J.; Lin, R.; Zhao, X. F. 2011. Grey relational analysis method for intuitionistic fuzzy multiple attribute decision making with preference information on alternatives, International Journal of Computational Intelligence Systems 4(2): 164-173. http://dx.doi.org/10.2991/ijcis.2011.4.2.5

$\mathrm{Xu}, \mathrm{Z}$. S. 2006. A note on linguistic hybrid arithmetic averaging operator in multiple attribute group decision making with linguistic information, Group Decision and Negotiation 15(6): 593-604. http://dx.doi.org/10.1007/s10726-005-9008-4

$\mathrm{Xu}, \mathrm{Z}$. S. 2010. Choquet integrals of weighted intuitionistic fuzzy information, Information Sciences 180(5): 726-736. http://dx.doi.org/10.1016/j.ins.2009.11.011

Yager, R. R. 2003. Induced aggregation operators, Fuzzy Sets and Systems 137(1): 59-69. http://dx.doi.org/10.1016/S0165-0114(02)00432-3

Yager, R. R. 2004a. Choquet aggregation using order inducing variables, International Journal of Uncertainty, Fuzziness and Knowledge-Based Systems 12(1): 69-88. http://dx.doi.org/10.1142/S0218488504002667

Yager, R. R. 2004b. OWA aggregation over a continuous interval argument with applications to decision making, Systems, Man, and Cybernetics, Part B: Cybernetics, IEEE Transactions on 34(5): 1952-1963. http://dx.doi.org/10.1109/TSMCB.2004.831154

Yin, M. S. 2012. Fifteen years of grey system theory research: a historical review and bibliometric analysis, Expert Systems with Applications, in press. http://dx.doi.org/10.1016/j.eswa.2012.11.002

Zadeh, L. A. 1965. Fuzzy sets, Information and Control 8(3): 338-353. http://dx.doi.org/10.1016/S0019-9958(65)90241-X

Zhang, X.; Liu, P. D. 2010. Method for aggregating triangular fuzzy intuitionistic fuzzy information and its application to decision making, Technological and Economic Development of Economy 16(2): 280-290. http://dx.doi.org/10.3846/tede.2010.18

Zhou, S. M.; Chiclana, F.; John, R.; Garibaldi, J. 2008. Type-1 OWA operators for aggregating uncertain information with uncertain weights induced by type-2 linguistic quantifiers, Fuzzy Sets and Systems 159(24): 3281-3296. http://dx.doi.org/10.1016/j.fss.2008.06.018

Zhu, J.; Hipel, K. W. 2012. Multiple stages grey target decision making method with incomplete weight based on multi-granularity linguistic label, Information Sciences 212(1): 15-32.

http://dx.doi.org/10.1016/j.ins.2012.05.011

Nian ZHANG is working for a doctor's degree at the School of Economic \& Management, Southwest Jiaotong University, China. He has contributed over several journal articles to professional journals, such as Applied Mathematical Modelling and Mathematical Problems in Engineering. His current research interests include decision-making theory, logistics engineering and management, and cooperative game theory. 